\title{
Erratum to: Associations between interhemispheric functional connectivity and the Automated Neuropsychological Assessment Metrics (ANAM) in civilian mild TBI
}

\author{
Chandler Sours • Joseph Rosenberg • Robert Kane • \\ Steve Roys • Jiachen Zhuo • \\ Kathirkamanthan Shanmuganathan • Rao P. Gullapalli
}

Published online: 2 July 2014

(C) Springer Science+Business Media New York 2014

Erratum to: Brain Imaging Behav (2014)

DOI 10.1007/s11682-014-9295-y

The original version of the article unfortunately contained errors. The details are as follows:

The online version of the original article can be found at http:/ dx.doi.org/10.1007/s11682-014-9295-y

C. Sours $\cdot$ J. Rosenberg $\cdot$ S. Roys $\cdot$ J. Zhuo $\cdot$ R. P. Gullapalli Magnetic Resonance Research Center, University of Maryland

School of Medicine, Baltimore, MD 21201, USA

C. Sours

e-mail: csour001@umaryland.edu

J. Rosenberg

e-mail: jrosenberg2@umm.edu

S. Roys

e-mail: sroys@umm.edu

J. Zhuo

e-mail: jzhuo@umm.edu

C. Sours $\cdot$ J. Rosenberg $\cdot$ S. Roys $\cdot$ J. Zhuo $\cdot$ K. Shanmuganathan $\cdot$ R. P. Gullapalli $(\bowtie)$

Department of Diagnostic Radiology \& Nuclear Medicine,

University of Maryland School of Medicine, Baltimore, MD 21201,

USA

e-mail: rgullapalli@umm.edu

K. Shanmuganathan

e-mail: kshanmuganathan@umm.edu

C. Sours · R. P. Gullapalli

Program in Neuroscience, University of Maryland School of

Medicine, Baltimore, MD 21201, USA

R. Kane

Georgetown University School of Medicine, Washington, DC 20057, USA

e-mail: kaner@georgetown.edu

Figures

Figure 5: The original Fig. 5 image and caption should be replaced with the below.

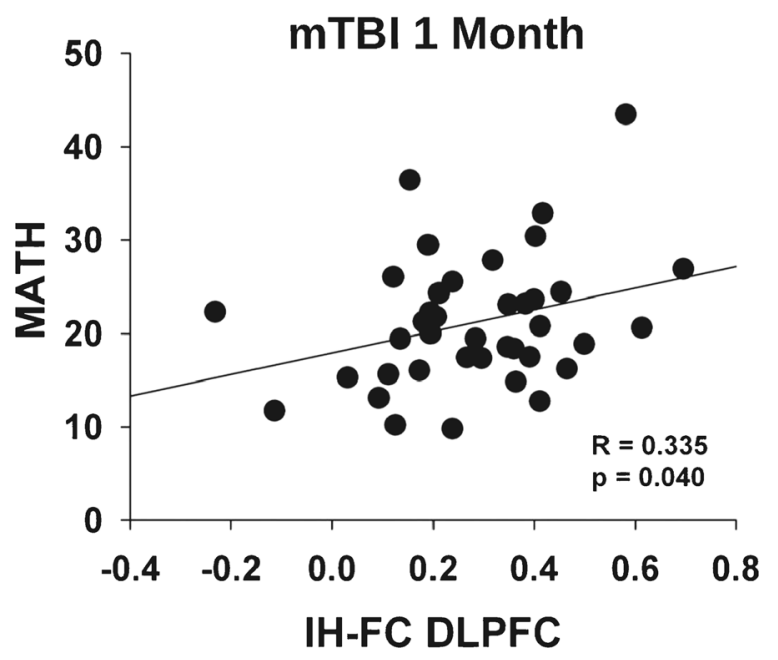

The Figure 5 legend should be replaced: "Correlation between rs-FC and the MATH subtest of the ANAM during 1 month follow up in mTBI participants using Pearson's partial correlations after correcting for age and years of education"

Tables

Table 5: The final row of Table 5 should be replaced with:

\begin{tabular}{rrrrrrr}
\hline WT-TH & $218.7+/$ & $220.2+/$ & $184.0+/$ & $200.5+/$ & 0.067 & 0.511 \\
& -46.4 & -46.9 & -41.3 & -36.2 & &
\end{tabular}


Table 6: The final row of Table 6 should be replaced with:

\begin{tabular}{rrrrrrr}
\hline WT-TH & $172.7+/$ & $188.3+/$ & $203.6+/$ & $221.6+/$ & 0.019 & 0.504 \\
& $-41 / 2$ & -32.8 & -34.7 & -32.8 & &
\end{tabular}

\section{Text}

1) In the 11th line of the abstract remove "and weighted throughput score $(p=0.001)$ " from sentence.

2) In the 16th line of the abstract replace " $p=0.009$ " with " $p=0.019$ "

3) In the 8th line of the second paragraph in the "ANAM battery" section of the Results, remove "and WT-TH $(p=0.001)$ " from sentence.
4) In the 16th line of the second paragraph under the "ANAM battery" section of the Results, replace " $(p=0.009)$ " with " $(p=0.019)$ "

5) In the 3rd line of the first paragraph under the "Correlations between imaging and ANAM" section of the Results, replace "at initial stage" with "at either time point"

6) In the 3rd-6th line of the first paragraph under the "Correlations between imaging and ANAM" section of the Results remove the sentence "However, at the one month follow up ..... WT-TH $(R=0.438, p=0.010)$ (Fig. 5)."

7) In the final line of the first paragraph under the "Correlations between imaging and ANAM" section of the Results, add reference "(Fig. 5)" to end of sentence after "1 month follow up".

8) In the 3rd line of the first paragraph under the "Neurocognitive performance" section of the Discussion, remove the phrase "as well as the WT-TH" 